\title{
Prospects for B Physics with the ATLAS and CMS Detectors
}

\author{
G. F. Tartarelli \\ I.N.F.N. - Sezione di Milano, via Celoria 16, 20135 Milano, Italia; \\ e-mail: Francesco.Tartarelli@mi.infn.it
}

\begin{abstract}
We describe the perspectives of the ATLAS and CMS experiments at the LHC in the B-physics field and review recent performance evaluations obtained by the two Collaborations. Despite having been designed for high- $p_{T}$ physics, both experiments have a rich B-physics programme and can compete with dedicated detectors in many studies of B-hadron production, decay and CP violation. These results can significantly contribute to the knowledge of these phenomena in the LHC era.
\end{abstract}

PACS: 12.15.Ff; 12.15.Hh; 13.20.He; $13.25 . \mathrm{Hw} ; 14.20 . \mathrm{Mr} ; 14.40 . \mathrm{Nd} ; 14.65 . \mathrm{Fy}$

\section{Introduction}

The LHC represents a unique opportunity for B-physics studies. Although the value is theoretically highly uncertain, the cross section for production of $b \bar{b}$ pairs is expected to be very high. Much higher than the $b \bar{b}$ production cross section at the $e^{+} e^{-} \mathrm{B}$ factories and about five times the cross section at the Tevatron running at $\sqrt{s}=2 \mathrm{TeV}$. Up to today (and also for the studies presented here) a value of $500 \mu \mathrm{b}$ has been assumed.

The ATLAS and CMS detectors are multi-purpose detectors optimized for Higgs and supersymmetry searches. However, their design is such that B-physics studies can be easily accommodated in their physics programmes.

Most of these studies are planned for the first period of low luminosity running when the LHC will not yet have reached the design luminosity of $10^{34} \mathrm{~cm}^{-2}$ $\mathrm{s}^{-1}$. During this period triggering and reconstruction of low $\mathrm{p}_{T}$ events, as required for B physics, will be easier. The exact luminosity and duration of this first run period is not known and will depend on the actual performance of the LHC, of course. In a first scenario an initial period of running at $10^{33} \mathrm{~cm}^{-2} \mathrm{~s}^{-1}$ was envisaged, with the luminosity then increasing linearly over a three-year period up to $10^{34} \mathrm{~cm}^{-2} \mathrm{~s}^{-1}$. More recently a new scenario with the LHC luminosity starting at $2 \times 10^{33} \mathrm{~cm}^{-2} \mathrm{~s}^{-1}$ and approaching the design luminosity after just one year has been proposed. The B-physics studies performed so far (including the ones presented here) still refer to the first of these scenarios and are going to be updated to the second one.

Even if a luminosity of $10^{33} \mathrm{~cm}^{-2} \mathrm{~s}^{-1}$ is called low in the LHC perspective, it is actually not so low at all: $\mathcal{O}\left(10^{12}-10^{13}\right) b \bar{b}$ pairs will be produced per year and per experiment. This represents a huge statistics limited only by the possibilities of the trigger and data acquisition systems. At this luminosity an additional 
average of 2.3 pile-up events from minimum bias superimposed to the interaction of interest must be taken into account in triggering and reconstruction. A much simpler and cleaner environment with respect to the high luminosity running where an average of 23 pile-up events are expected. For some channels (rare B decays, see below) the possibility to continue these studies at high luminosity has been demonstrated.

As it will be described below, precision measurements are possible by ATLAS and CMS in several channels: thanks to the high statistics, the precision achievable is better than at Tevatron and at the $e^{+} e^{-} \mathrm{B}$ factories and is in some cases competitive with LHCb, the LHC experiment dedicated to B physics. The higher luminosity in ATLAS and CMS with respect to LHCb $\left(2 \times 10^{32} \mathrm{~cm}^{-2}\right.$ $\mathrm{s}^{-1}$ ) implies that in some channels precision measurements can be done by ATLAS and CMS just after few months or one year of running. So that even if the ultimate precision achievable on some measurements might be better for $\mathrm{LHCb}$ running more time at a suitable luminosity ( $\mathrm{LHCb}$ will keep locally at its interaction point the luminosity at its preferred value for the experiment lifetime), first precise measurements can come from both ATLAS and CMS. Moreover, these measurements can provide cross-checks of the $\mathrm{LHCb}$ results as they will be affected by different systematics and can be combined with them so enhancing the overall LHC precision.

Not all B-physics possibilities of ATLAS and CMS have been assessed yet. Some benchmark channels have been looked in great detail, using full detector simulation and sophisticated analysis method. Some of these results are summarized here. The programme will evolve with time: the two experiments will follow the theoretical developments in the field and look at the results that the $\mathrm{B}$ factories and the Tevatron experiments will obtain in the next years.

Results presented here are mostly taken from the ATLAS Detector and Physics Performance Technical Design Report [1] and from the final report of the Workshop on the Standard Model Physics (and more) at the LHC [2] held at CERN in 1999, for both ATLAS and CMS. Also LHCb numbers (used for comparison of the performances in some cases) are taken from this last reference (updated numbers - if available - can be found in [3]).

\section{ATLAS and CMS Characteristics for B-physics}

Several characteristics of the ATLAS and CMS designs are well suited for Bphysics studies. Good secondary vertex resolution is guaranteed by the presence of pixel detector layers (three layers in the barrel region) close to the interaction point. In ATLAS low- $p_{T}$ muon identification down to $3 \mathrm{GeV} / \mathrm{c}$ is possible thanks to the combined use of the muon spectrometer and of the hadronic calorimeter. Low- $p_{T}$ electron identification down to about $0.5 \mathrm{GeV} / \mathrm{c}$ is done by a transition radiation detector integrated with the outer straw tracker system (the Transition Radiation Tracker or TRT) and by the calorimeters. Moreover, the TRT is used to trigger (at Level 2) on $J / \psi \rightarrow e^{+} e^{-}$. In CMS low- $p_{T}$ muon identification is done by the muon spectrometer and low- $p_{T}$ electron identification is possible thanks to a high resolution crystal calorimeter together with the tracking system. 
Table 1. Indicative efficiencies and dilutions for flavour tagging algorithms studied by ATLAS and CMS. The first two rows refer to Opposite Side (OS) tagging algorithms and the remaining ones to Same Side (SS) algorithms.

\begin{tabular}{lcc}
\hline & $\epsilon(\%)$ & $\mathrm{D}$ \\
\hline lepton (OS) & $2-3$ & $0.4-0.5$ \\
jet charge (OS) & 65 & 0.18 \\
$B-\pi$ (SS) & 82 & 0.16 \\
jet charge (SS) & 21 & 0.32 \\
jet charge (SS) & 50 & 0.23 \\
\hline
\end{tabular}

The stronger B field in CMS than in ATLAS (4 T with respect to $2 \mathrm{~T}$ ) has the consequence that a better mass resolution is achievable.

Neither ATLAS nor CMS have dedicated detectors for hadron identification. However, in ATLAS a limited $\pi / K$ separation is achieved indirectly by using $d E / d x$ energy loss in the straw tracker. The separation obtained is of the order of 1.0-1.5 $\sigma$ (it is $p_{T}$ dependent) and, used on a statistical basis, has proven to be very useful in some of the analyses described below.

Many of the flavour tagging algorithms developed in the past years by the LEP experiments and by CDF appear feasible in ATLAS and CMS, too. Indicative efficiencies and dilutions are summarized in Table 1.

Both algorithms based on the flavour tagging of the B which has decayed in the channel under investigation (same-side tagging) and algorithms based on tagging the other $\mathrm{b}$ quark in the event (opposite-side tagging) have been studied. Opposite-side algorithms include lepton tagging or jet charge tagging. Same-side tagging is performed by jet charge tagging or by studying pions correlated with the $\mathrm{b}$ (or the $\bar{b}$ ) quark through $B^{\star \star}$ decays (CMS) or through fragmentation (ATLAS). Preliminary work has also been done to combine these algorithms but a full combination on an event-by-event basis of all algorithms taking into account their efficiencies, dilutions and other effects still needs to be done.

It must also be considered that the period in which B-physics studies will be performed corresponds to the initial one in which both Collaborations do not plan to have the full detector in place as it has been decided to defer the installation of some components to a later time. For example, both detectors will have one less pixel layer initially but this will not be the innermost layer which is the most crucial for B-physics. Other detectors that might be staged are some forward components of the muon system in both experiments and the very forwards part of the TRT in ATLAS.

\section{B-physics Triggers}

Triggering on B-physics events is a major issue at the LHC. One has to select events with relatively low $\mathrm{p}_{T}$ and with a complex signature from the enormous $p p$ interaction rate. 
In both ATLAS and CMS the rate reduction from the $40 \mathrm{MHz}$ bunch crossing rate to the $\mathcal{O}(100) \mathrm{Hz}$ rate which can be written to tape is achieved with a multilevel trigger system.

At the lowest trigger level (LVL1 in both experiments), the selection is based on leptonic signatures from $b$ decays. In ATLAS a muon with $p_{T}>6 \mathrm{GeV} / \mathrm{c}$ is required. A dimuon trigger with lower threshold $\left(p_{T} \sim 3 \mathrm{GeV} / \mathrm{c}\right)$ seems also feasible and will be included in future results. ATLAS triggers are discussed in detail in [4]. In CMS both single and dilepton triggers (with the possibility to use $\eta$ and $p_{T}$ dependent cuts) are feasible. For the studies obtained in [2] and reported here, the following triggers were assumed: one $\mu$ with $p_{T}>7 \mathrm{GeV} / \mathrm{c}$, two $\mu$ 's with $p_{T}>2-4 \mathrm{GeV} / \mathrm{c}$ ( $\eta$ dependent), one electron with $p_{T}>12 \mathrm{GeV} / \mathrm{c}$, two electrons with $p_{T}>5 \mathrm{GeV} / \mathrm{c}$, one electron with $p_{T}>5 \mathrm{GeV} / \mathrm{c}$ plus one $\mu$ with $p_{T}>4 \mathrm{GeV} / \mathrm{c}$. Up-to-date CMS trigger menus may be found in [5].

After the LVL1 selection the rate of production of $b \bar{b}$ pairs is still high. For example, in ATLAS the $b \bar{b}$ cross section is still about $2.3 \mu \mathrm{b}$, which corresponds to the initial selection of about $2 \times 10^{10} b \bar{b}$ pairs per year.

The selection of specific channels is done at next trigger levels: the feasibility has been demonstrated for some exclusive and semi-exclusive channels, including the ones described below. The trigger systems of both experiments are flexible enough to accommodate new triggers as physics needs will change with time.

Neither ATLAS nor CMS have purely hadronic triggers at LVL1 (the situation is different for $\mathrm{LHCb}$ which can count on purely hadronic triggers at its lowest trigger level). This causes a reduction of statistics in studies of fully hadronic B decays.

\section{$4 \mathrm{~J} / \psi$ Samples}

As for the Tevatron experiments during Run I, events containing a $J / \psi$ will represent a large source of B events. Large and clean samples of both $J / \psi \rightarrow \mu^{+} \mu^{-}$ and $J / \psi \rightarrow e^{+} e^{-}$will be available in ATLAS and CMS. Typical mass resolutions for $J / \psi \rightarrow \mu^{+} \mu^{-}$are about $40 \mathrm{MeV}$ in ATLAS and $20 \mathrm{MeV}$ in CMS. For $J / \psi \rightarrow e^{+} e^{-}$, ATLAS estimates a resolution of about $60 \mathrm{MeV}$ when fitting only the core of the distribution, which has a long tail towards low masses due to bremsstrahlung energy losses. Good performance is expected in the reconstruction of inclusive $b \rightarrow J / \psi X$ events, also at high $p_{T}$ (greater than $50 \mathrm{GeV}$ ), that's to say inside dense and collimated jets, which are useful for production studies. Examples of B decay channels containing a $J / \psi$ in the final state studied so far are the following: $B_{d, s}^{0} \rightarrow J / \psi K_{s}^{0}, B_{s}^{0} \rightarrow J / \psi \phi, B_{d}^{0} \rightarrow J / \psi K^{\star 0}, B^{+} \rightarrow J / \psi K^{+}$, $B_{c}^{+} \rightarrow J / \psi \pi^{+}, B_{d, s}^{0} \rightarrow J / \psi \eta, \Lambda_{b} \rightarrow J / \psi \Lambda^{0}$ and inclusive $b \rightarrow J / \psi X$ samples.

Some of these channels are briefly described in this section.

\section{$4.1 \quad B_{d, s}^{0} \rightarrow J / \psi K_{s}^{0}$}

Large and clean samples of $B_{d}^{0} \rightarrow J / \psi K_{s}^{0}$ will be available in ATLAS and CMS. It is expected that about $165 \mathrm{k}$ events will be collected by ATLAS in one year and about 450k events/year by CMS: the higher CMS yield is due to the presence 

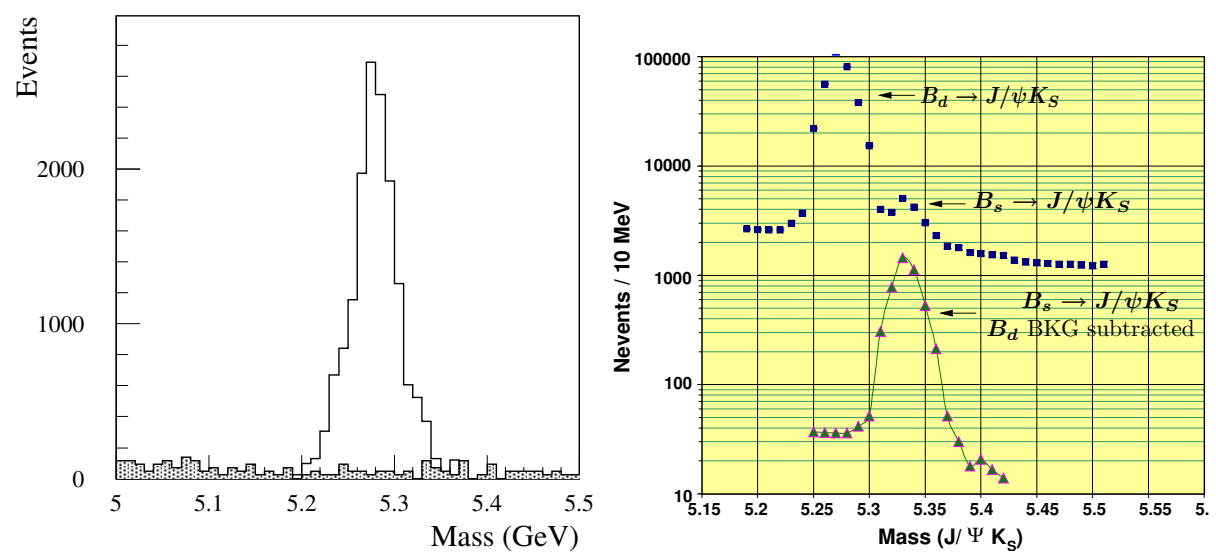

Fig. 1. ATLAS (left) and CMS (right) mass peaks for $B_{d}^{0} \rightarrow J / \psi K_{s}^{0}$ with superimposed the expected background level. For CMS the $B_{s}^{0}$ peak with and without the neighbour $B_{d}^{0}$ peak subtracted is also shown.

of a low- $p_{T}$ dimuon trigger as explained in Sect. 3. The reconstructed $B_{d}^{0}$ mass peaks are shown in Fig. 1.

This channel is universally considered the golden channel to measure the angle $\beta$ of the unitary triangle of the Cabibbo-Kobayashi-Maskawa (CKM) quark mixing matrix. In fact, to a very good approximation this decay is dominated by only one CKM amplitude in the Standard Model and the time-dependent CP asymmetry can be expressed as: $A_{C P}^{m i x}=-\sin \phi_{d}=-\sin 2 \beta$, where $\phi_{d}$ is the $B_{d}^{0}-\overline{B_{d}^{0}}$ weak mixing phase. The large number of events available in ATLAS and CMS implies an excellent sensitivity to the measurement of $\sin 2 \beta$ : after just one year of data taking, a precision of $\sigma(\sin 2 \beta)=0.017$ for ATLAS and $\sigma(\sin 2 \beta)=0.015$ for CMS can be obtained. That is to say a first measurement of $\sin 2 \beta$ with a precision at the level of 0.015 might be obtained in relatively short time: such a precision appears to be out of reach by the $e^{+} e^{-}$B-factories and the Tevatron experiments (a combined uncertainties of about 0.03 is projected) by the time the LHC starts. For comparison, $\mathrm{LHCb}$ expects $90 \mathrm{k}$ events and a corresponding precision of $\sigma(\sin 2 \beta)=0.021$ after one year of data taking.

The direct component of the time-dependent $\mathrm{CP}$ asymmetry in this decay, $A_{C P}^{d i r}$, is expected to be very small in the Standard Model. It is interesting to check this Standard Model prediction. In a study performed by ATLAS [6], it has been estimated that a $5 \sigma$ discovery can be obtained if $A_{C P}^{d i r}$ is at least 0.10 and in case of a null result values of $A_{C P}^{d i r}$ greater than 0.04 can be excluded at the $95 \%$ CL after three years of running at low luminosity.

CMS has also studied the possibility to reconstruct the decay $B_{s}^{0} \rightarrow J / \psi K_{s}^{0}$. The reconstruction is more difficult than the $B_{d}^{0} \rightarrow J / \psi K_{s}^{0}$ channel: the $\sigma \cdot B R$ is smaller (it has been assumed that it is an order of magnitude smaller than the $B_{d}^{0}$ counterpart), the $\mathrm{S} / \mathrm{B}$ comes out smaller and good mass resolution is needed to separate the $B_{s}^{0}$ peak from the more pronounced $B_{d}^{0}$ peak which is only about $90 \mathrm{MeV}$ apart. As explained in Sec. 2 the strong B field in CMS provides the 
Table 2. ATLAS and CMS relative statistical uncertainties on $\Delta \Gamma_{s}, \Gamma_{s}$ and absolute uncertainty on $\phi_{s}$ for two different values of $x_{s}$ after three years of running at low luminosity.

\begin{tabular}{lcccc}
\hline & $\Delta \Gamma_{s}$ & $\Gamma_{s}$ & $\phi_{s}\left(x_{s}=20\right)$ & $\phi_{s}\left(x_{s}=40\right)$ \\
\hline ATLAS & $12 \%$ & $0.7 \%$ & 0.03 & 0.05 \\
CMS & $8 \%$ & $0.5 \%$ & 0.014 & 0.03 \\
\hline
\end{tabular}

mass resolution necessary to separate the two peaks. An annual event yield of $4.1 \mathrm{k}$ events with a $\mathrm{S} / \mathrm{B}$ of about 0.5 has been estimated. The reconstructed mass peak is shown in Fig. 1.

\section{$4.2 \quad B_{s}^{0} \rightarrow J / \psi \phi$}

Large and clean samples of $B_{s}^{0} \rightarrow J / \psi \phi$ decays will also be available in ATLAS and CMS. It is estimated that ATLAS will reconstruct about $100 \mathrm{k}$ of these events (untagged) in one year of data taking and CMS will reconstruct about twice this number (with S/B of about 6 and 10, respectively). ATLAS plans to increase the statistics by the inclusion of a low- $p_{T}$ dimuon trigger. For comparison, CDF expects $6 \mathrm{k}$ events in $2 \mathrm{fb}^{-1}$ and $\mathrm{LHCb}$ expects $74 \mathrm{k}$ events/year.

This decay may be used to probe the $B_{s}^{0}-\overline{B_{s}^{0}}$ weak mixing phase $\phi_{s}=$ $-2 \delta \gamma=-2 \lambda^{2} \eta$ (where $\delta \gamma$ is an angle related to the other non-squashed unitary triangle of the CKM matrix and $\lambda$ and $\eta$ are Wolfenstein parameters). However, the $J / \psi \phi$ final state in this decay is an admixture of different $\mathrm{CP}$ eigenstates and an angular analysis of the decay products $J / \psi\left(\mathrm{l}^{+} \mathrm{l}^{-}\right) \phi\left(\mathrm{K}^{+} \mathrm{K}^{-}\right)$is needed. In principle, eight independent parameters might be extracted by this analysis. However, as it was done in [2] because of the experimental precision available, one can extract only $\phi_{s}$, two unknown amplitudes $\left|A_{\perp}(0)\right|$ and $\left|A_{\|}(0)\right|$, the decay rate difference $\Delta \Gamma_{s}=\Gamma_{H}-\Gamma_{L}$ and the mean decay rate $\Gamma_{s}=\left(\Gamma_{H}+\Gamma_{L}\right) / 2$ of the mass eigenstates $B_{H}^{0}$ and $B_{L}^{0}$ and fix the remaining parameters: two strong phases $\delta_{1}$ and $\delta_{2}$ and the mass difference $\Delta M_{s}$ (which can be measured independently: see Sect. 6.3). The precisions available after three years of running at low luminosity on some of these parameters is summarised in Table 2.

For comparison, after five years of data taking $\mathrm{LHCb}$ will achieve a $9 \%$ statistical uncertainty on $\Delta \Gamma_{s}$, a $0.6 \%$ uncertainty on $\Gamma_{s}$ and a 0.02 uncertainty on $\phi_{s}$ (for $x_{s}=20$ ). These sensitivities might not be enough to measure $\phi_{s}$ which is expected to be very small in the Standard Model: $\phi_{s}=\mathcal{O}(0.03)$. However, the smallness of this phase makes this decay an ideal place to look for new physics effects. Indeed, several scenarios beyond the Standard Model foresee $\mathrm{CP}$-violating contribution effects in this decay.

\section{$4.3 \quad B_{d, s}^{0} \rightarrow J / \psi \eta$}

The $B_{s}^{0} \rightarrow J / \psi \eta$ channel is similar to the $B_{s}^{0} \rightarrow J / \psi \phi$ channel. However, the final state is now a $\mathrm{CP}$ eigenstate and the $\mathrm{CP}$-violating asymmetry has a simpler 

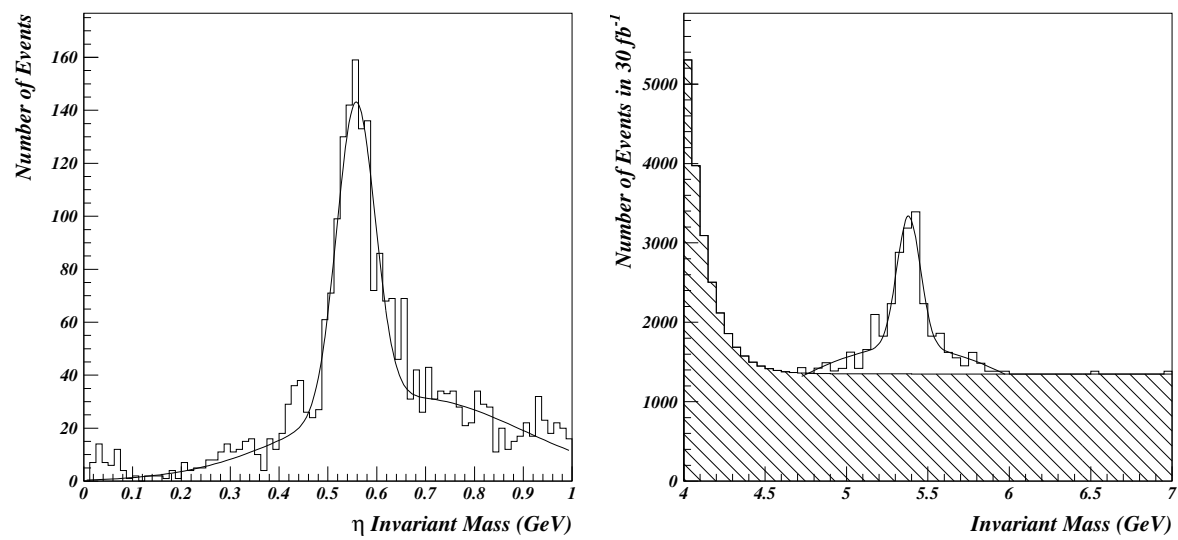

Fig. 2. ATLAS mass peaks for $\eta \rightarrow \gamma \gamma$ (left) and $B_{s}^{0} \rightarrow J / \psi \eta$ (right) after three years of running at low luminosity.

relation with the weak mixing phase: $A_{C P}^{m i x}=\sin \phi_{s}$. Again the asymmetry is expected to be very small but new physics effects can substantially enhance it. The analysis is complicated by the difficulty to identify low- $p_{T}$ photons in the calorimeter to reconstruct the $\eta \rightarrow \gamma \gamma$ decay. An ATLAS study [7] [8] estimates that about 10k events can be reconstructed after three years of running at low luminosity with a S/B of about 1 . The $\eta \rightarrow \gamma \gamma$ peak reconstructed in the ATLAS calorimeter and the $B_{s}^{0}$ peak with superimposed the expected background level are shown in Fig. 2.

With this yield it has been estimated that the uncertainty on $\sin \phi_{s}$ will be of $\sigma\left(\sin \phi_{s}\right)=0.27$ for $x_{s}=19$ and $\sigma\left(\sin \phi_{s}\right)=0.31$ for $x_{s}=30$ after three years of running at low luminosity.

ATLAS has also studied the channel $B_{d}^{0} \rightarrow J / \psi \eta$ but its reconstruction does not appear to be feasible.

\section{4 b-production studies}

Large samples of exclusive and inclusive b-hadron decays will be available to perform beauty production studies. These studies include determination of the total and differential single b-quark cross section and studies of $b \bar{b}$ correlations (relative azimuthal distance, pair rapidity, invariant mass and so on). These studies will extend those possible at Tevatron with larger statistics, at higher $\sqrt{s}$, and at higher b-quark $p_{T}$. Moreover, the ATLAS and CMS measurements will complement those made by $\mathrm{LHCb}$ which operates in a different region of the phase space.

Exclusive decays containing a $J / \psi$, which are also used for B-properties and $\mathrm{CP}$ violation studies (as briefly summarised in previous sections), can be also used for production studies at low b-quark $p_{T}$. Inclusive $J / \psi X$ decays can be used for $p_{T}$ from about $50 \mathrm{GeV}$ up to $\sim 300 \mathrm{GeV}$. To explore even higher transverse momentum regions, a different technique exploiting inclusive b-jet tagging 
(using impact parameter information to discriminate b quarks from gluon and light quark jets) may be used.

Due to the large statistics available, $b \rightarrow J / \psi X$ with $J / \psi \rightarrow \mu \mu$ can also be used for $b \bar{b}$ correlation studies, like the azimuthal separation, $\Delta \phi_{b \bar{b}}$, between the $b \bar{b}$ pair. One can, in fact, study the separation between the $J / \psi$ and another $\mu$ in the event, assuming they are coming from the decay of the $\mathrm{b}$ and $\bar{b}$ of the event. This is an alternative to the usual study of the separation between two muons, made possible by the unprecedented statistics available at the LHC. These three-muon events are cleaner than dimuon events which are affected by higher background, especially at low $p_{T}$, dominated by $\pi / K$ decays in flight. Softer cuts can be applied in three muons events so avoiding to depopulate the crucial low $\Delta \phi_{b \bar{b}}$ region which is the most interesting to understand the QCD production mechanism of these events.

\section{Dimuon Samples}

Samples of dimuon events collected outside the $J / \psi$ and $\psi \prime$ resonances can be used to look for some rare B decays. Channels looked at so far are the following ones: $B_{d, s}^{0} \rightarrow \mu^{+} \mu^{-}, B_{d}^{0} \rightarrow K^{\star 0} \mu^{+} \mu^{-}, B_{d}^{0} \rightarrow \rho^{0} \mu^{+} \mu^{-}, B_{s}^{0} \rightarrow \phi \mu^{+} \mu^{-}$.

These are all rare decays, with Standard Model branching ratios less than $10^{-5}$, which proceed through flavour changing neutral currents loops. These loops are sensitive to new physics effects which can also substantially enhance the branching ratios. Information on masses and couplings of new particles participating in the loops might be deduced.

\section{$5.1 \quad B_{d, s}^{0} \rightarrow \mu^{+} \mu^{-}$}

The decays $B_{d}^{0} \rightarrow \mu^{+} \mu^{-}$and $B_{s}^{0} \rightarrow \mu^{+} \mu^{-}$are self-triggering modes and easy to reconstruct as only two muons are involved. Indeed it has been shown by both Collaborations that the study of these decays can be continued beyond the low-luminosity running period, when the luminosity will reach $10^{34} \mathrm{~cm}^{-2} \mathrm{~s}^{-1}$.

In these studies the Standard Model branching ratios of $3.5 \times 10^{-9}$ and $1.5 \times 10^{-10}$ for $B_{s}^{0} \rightarrow \mu^{+} \mu^{-}$and $B_{d}^{0} \rightarrow \mu^{+} \mu^{-}$, respectively, have been assumed. In this hypothesis it has been estimated that after three years of running at low luminosity and one year of running at high luminosity, both ATLAS and CMS should be able to make a clear $5 \sigma$ observation of the decay $B_{s}^{0} \rightarrow \mu^{+} \mu^{-}$. The observation of the $B_{d}^{0} \rightarrow \mu^{+} \mu^{-}$appears more difficult as only an handful of events will be available.

\section{$5.2 \quad B_{d}^{0} \rightarrow K^{\star 0} \mu^{+} \mu^{-}$}

The decay $B_{d}^{0} \rightarrow K^{\star 0} \mu^{+} \mu^{-}$is also easy to trigger on and to reconstruct. Assuming a Standard Model branching ratio of $1.5 \times 10^{-6}$, ATLAS expects to reconstruct about 700 events/year and CMS expects $4.2 \mathrm{k}$ events/year (with a S/B of about 7 and 9, respectively). Again the higher CMS yield is due to the low- $p_{T}$ dimuon trigger: the ATLAS yield will increase with the inclusion of a 


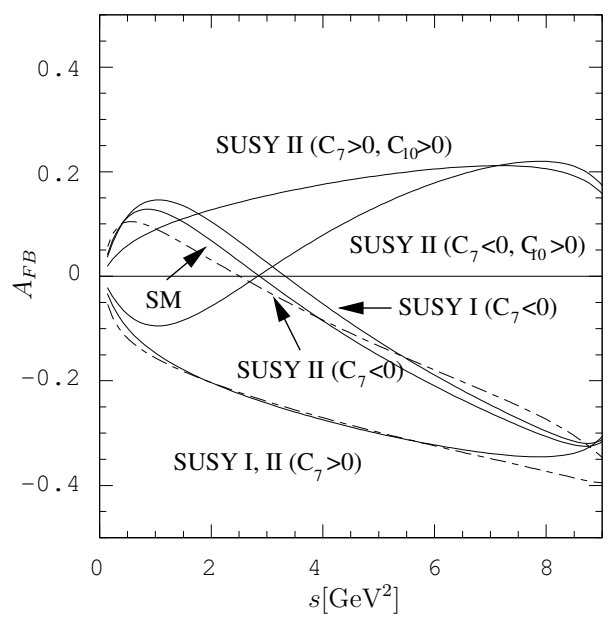

Fig. 3. Lepton forward-backward asymmetry in $B_{d}^{0} \rightarrow K^{\star 0} \mu^{+} \mu^{-}$decay as a function of the squared dimuon mass $s$. The asymmetry is shown for the standard model prediction and for two SUSY models.

similar trigger. The large number of events available will allow detailed studies of this decay. Indeed, the lepton forward-backward asymmetry in this decay can distinguish between Standard Model and some SUSY models (see Fig. 3).

ATLAS estimates a $5 \%$ precision on $A_{F B}$ after 3 years of running at low luminosity. The corresponding CMS precision on this measurement is under evaluation. For comparison, LHCb estimated $4.5 \mathrm{k}$ events/year for this decay (see also [3] for an update of this analysis) and a $2.4 \%$ precision on the asymmetry after 1 year of data taking.

\section{Hadronic Samples}

Samples of fully hadronic B decays have also been studied by ATLAS and CMS. As explained in Sect. 3 neither ATLAS nor CMS have fully hadronic trigger at the lowest trigger level. This implies that at least one lepton will always be present in these events. This causes a reduction of the yield. However, when flavour tagging is needed in these events, the trigger lepton can be used as lepton tag from the decay $b \rightarrow l$ of the other $b$ quark in the event.

Some of the hadronic channels studied so far are described below.

\section{1 $\quad B_{d}^{0} \rightarrow \pi^{+} \pi^{-}$}

The reconstruction of the $B_{d}^{0} \rightarrow \pi^{+} \pi^{-}$signal in ATLAS and CMS is made difficult by the absence of dedicated $\pi / K$ separation detectors to reduce the dangerous backgrounds from other two-body B decays. ATLAS has developed a likelihood method in which the likelihood of a given decay hypothesis is calculated using all the available information: the proper time (and its uncertainty), 

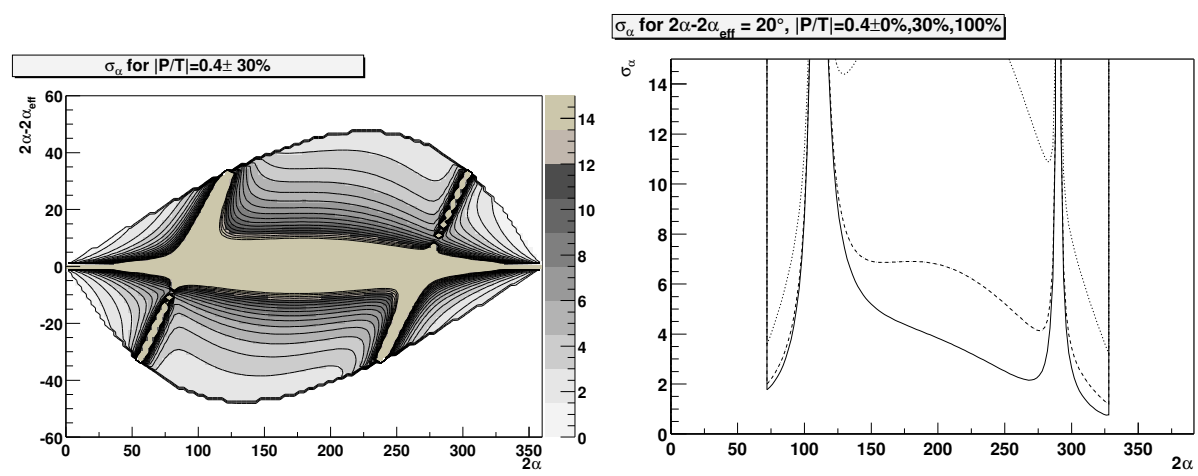

Fig. 4. Sensitivity on $\alpha$ in the plane $\left(2 \alpha, 2 \alpha-2 \alpha_{\text {eff }}\right)$ assuming that $|P / T|=0.4$ and that its value is known with a $30 \%$ uncertainty (left). Sensitivity on $\alpha$ as a function of $2 \alpha$ for fixed values of $2 \alpha-2 \alpha_{\text {eff }}=20^{\circ}$ and $|P / T|=0.4$ (right). The three curves correspond to three different assumed uncertainties on $|P / T|$ : $0 \%$ (solid), $30 \%$ (dashed) and $100 \%$ (dotted). These plots refer to the expected combined LHC performance (LHCb included).

the reconstructed mass, the initial state flavour and the $d E / d x$ estimate, using the statistical $\pi / K$ separation described in Sect. 2. Roughly the yield is about $2.3 \mathrm{k}$ events/year with a S/B of the order of 0.2 with respect to the two body background and $\mathrm{S} / \mathrm{B} \sim 1.6$ with respect to the other background (in $\pm 1 \sigma$ about the nominal $B_{d}^{0}$ mass). However, as explained, the technique is much more powerful than a simple event counting. The unbinned likelihood fit allows the extraction of the $\mathrm{CP}$ violating asymmetries $A_{C P}^{d i r}, A_{C P}^{m i x}$ and of their correlation coefficient. The expected sensitivities on each of these three parameters are: $\sigma\left(A_{C P}^{\text {dir }}\right)=0.16, \sigma\left(A_{C P}^{\text {mix }}\right)=0.21$ and $\sigma^{\text {corr }}=-0.25$. Numbers from CMS are under revaluation. Clearly the absence of hadronic triggers and of a dedicated detector for hadron identification reduces the performance in this channel and indeed $\mathrm{LHCb}$ estimates a yield of $4.9 \mathrm{k}$ events/year (with a $\mathrm{S} / \mathrm{B}$ of about $15)$ and better sensitivities on the $\mathrm{CP}$ violating asymmetries $\left(\sigma\left(A_{C P}^{d i r}\right)=0.09\right.$, $\sigma\left(A_{C P}^{m i x}\right)=0.07$ after one year of data taking).

This decay can be used to measure the angle $\alpha$ of the CKM matrix. However its extraction is not at all straightforward because of the presence of penguin contributions. An $\alpha_{\text {eff }}$ parameter (which would reduce to $\alpha$ in the absence of penguin contributions) can be introduced and extracted together with $\alpha$ from two relations containing the two $\mathrm{CP}$ violation asymmetries and $|P / T|$, the ratio of the penguin to tree amplitude of the process (the assumption that $\alpha=\pi-(\beta+\gamma)$ needs also to be made) [9]:

$$
\begin{aligned}
A_{C P}^{\text {mix }} & =-\sqrt{1-A_{C P}^{\text {dir }}{ }^{2}} \sin 2 \alpha_{\text {eff }} \\
\cos \left(2 \alpha-2 \alpha_{e f f}\right) & =\frac{1}{\sqrt{1-A_{C P}^{\text {dir }} 2}}\left[1-\left(1-\sqrt{1-A_{C P}^{\text {dir }}{ }^{2}} \cos 2 \alpha_{e f f}\right)\left|\frac{P}{T}\right|^{2}\right]
\end{aligned}
$$

However, the theoretical calculation of $|P / T|$ is extremely difficult and is 


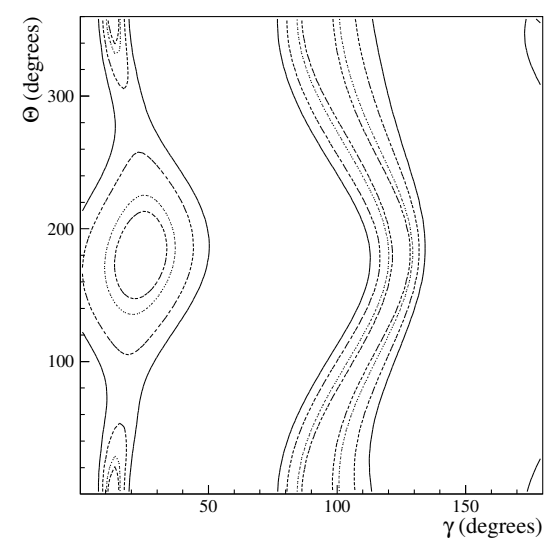

Fig. 5. ATLAS sensitivity to $\gamma$ as a function of $\gamma$ and of the hadronic phase $\theta$ (for fixed amplitude $d=0.3$ ) after three years of running at low luminosity. Contour lines correspond to sensitivities of $8^{\circ}$ (solid), $10^{\circ}$ (dashed) $15^{\circ}$ (dotted) and $20^{\circ}$ (dash-dotted).

difficult to foresee with which precision this value will be known at the time of the LHC start. Indeed, the precision on $\alpha$ depends strongly on the value of $\alpha$ itself, on $\alpha_{e f f}$, on $|P / T|$ and on its error $\sigma(|P / T|)$. This has the consequence that it is difficult to quote the uncertainty on $\alpha$ as a single value but it is rather better to show contour plots in the parameter space. As an example of the dependence on these parameters, in Fig. 4 it is shown $\sigma(\alpha)$ as a function of $2 \alpha$ and $2 \alpha-2 \alpha_{e f f}$ for a fixed value of $|P / T|$ and also the degradation of $\sigma(\alpha)$ as the error on $|P / T|$ increases. These plots have been obtained for the expected LHC combined precision on the $\mathrm{CP}$ violating asymmetries. A large variation of the result depending on the value of the various parameters can be clearly seen.

\section{2 $\quad B^{0} \rightarrow h^{+} h^{-}$}

The four CP asymmetries in $B_{d}^{0} \rightarrow \pi^{+} \pi^{-}$and $B_{s}^{0} \rightarrow K^{+} K^{-}$can be related to the angle $\gamma$ of the CKM matrix, to $\phi_{d}=2 \beta$ and to an hadronic amplitude $d$ and phase $\theta$ (assuming U-spin symmetry). The four unknowns can be extracted simultaneously. The decay $B_{d}^{0} \rightarrow \pi^{+} \pi^{-}$has already been discussed in the previous section. The $B_{s}^{0} \rightarrow K^{+} K^{-}$is studied simultaneously to the $B_{d}^{0} \rightarrow \pi^{+} \pi^{-}$ decay and the two $\mathrm{CP}$ asymmetries for this decay are extracted from the same likelihood fit.

The sensitivity obtainable on $\gamma$ depends on the values of the other parameters (including $\gamma$ itself) and can be illustrated as contour lines in the $\gamma-\theta$ plane (see Fig. 5).

For the present value favoured by CKM fits [10], $\gamma \approx 60^{\circ}$, a precision of $\sigma(\gamma) \sim 8^{\circ}$ after 3 years of running at low luminosity has been estimated in ATLAS. CMS numbers for this analysis are under revaluation. 


\section{3 $\quad B_{s}^{0} \rightarrow D_{s}^{+} \pi^{-}$}

The decay $B_{s}^{0} \rightarrow D_{s}^{+} \pi^{-}$with $D_{s}^{+} \rightarrow \phi \pi^{+}$can be used to measure the mixing parameter $\Delta M_{s}$. ATLAS also uses the decay $B_{s}^{0} \rightarrow D_{s}^{+} a_{1}^{-}$with $D_{s}^{+} \rightarrow \phi \pi^{+}$and $a_{1}^{-} \rightarrow \rho_{0} \pi^{-} \rightarrow \pi^{+} \pi^{-} \pi^{-}$. These are exclusive channels and so good resolution is achievable on the $B_{s}^{0}$ proper time to resolve its rapid oscillations: ATLAS estimates a proper time resolution of about $\sigma(\tau)=71 \mathrm{fs}$ and CMS of $\sigma(\tau)=$ 65 fs. A degradation of about $20 \%$ is expected for the ATLAS number as a consequence of the recent decision to increase the radius of the innermost pixel layer from 43 to $50.5 \mathrm{~mm}$ because of the increase of the beam pipe outer diameter from 50 to $69.2 \mathrm{~mm}$ : the exact impact on the proper time resolution and on the $B_{s}^{0}$ mixing analysis is under evaluation.

Large samples of these decays will be available: about 3.5k events/year in ATLAS and about 4.5k events/year in CMS. As explained in Sect. 3, the Level 1 trigger request implies that there will always be one lepton in these events that can be used to tag the flavour of these events through the lepton tagging method. After one year of data taking at low luminosity, both experiments would have a $95 \%$ CL sensitivity up to $\Delta M_{s}$ of about $40 \mathrm{ps}^{-1}$. This is well above the allowed range of the Standard Model. Indeed if no new physics effects appear and indications coming from experimental searches [11] [12] and present fits to the CKM matrix [10] are correct, $\Delta M_{s}$ shouldn't be much larger than about 20 $\mathrm{ps}^{-1}$. In this hypothesis the Tevatron experiments should be able to observe the $B_{s}^{0}$ oscillations before the LHC start. In this scenario it might be more interesting to evaluate the precision available on a $\Delta M_{s}$ measurement assuming mixing as already been observed: for $\Delta M_{s}=20 \mathrm{ps}^{-1}$ an uncertainty of $\sigma\left(\Delta M_{s}\right)=0.11$ has been estimated. For comparison, better sensitivity (up to $\Delta M_{s} \sim 58 \mathrm{ps}^{-1}$ ) can be obtained by LHCb thanks to a higher event yield (about 34.5k tagged events/year, due to the hadronic triggers) and a better proper time resolution (about $43 \mathrm{fs}$ ).

\section{Conclusions}

The LHC experiments can significantly contribute to the knowledge of B physics and $\mathrm{CP}$ violation due to the unprecedented statistics available. The LHCb detector has been optimized for this kind of studies and, indeed, has unique capabilities in some channels. However, in many channels ATLAS and CMS have performances comparable to $\mathrm{LHCb}$ and can give a substantial contribution to the overall LHC performance. A brief and incomplete summary of these studies, including some new results, has been given here.

\section{Acknowledgements}

The work contained in this paper is the result of the effort of many members of the ATLAS and CMS B-physics groups. The author wishes to thank Daniel Denegri, Nick Ellis, Fabiola Gianotti and Yves Lemoigne for their help. 


\section{References}

1. The ATLAS Collaboration, ATLAS Detector and Physics Performance Technical Design Report, CERN/LHCC/99-14/15 (1999).

2. G. Altarelli, M. L. Mangano (Eds.), Proceedings of the Workshop on the Standard Model Physics (and More) at the LHC, CERN 2000-004 (2000).

3. P. Koppenburg, these proceedings.

4. S. George, these proceedings.

5. S. Dasu, these proceedings.

6. P. A. Booth et al., ATLAS Note, ATL-PHYS-2001-013.

7. F. Ohlsson-Malek and M. Melcher, ATLAS Note, ATL-PHYS-2001-023.

8. C. Driouichi et al., ATLAS Communication, ATL-COM-PHYS-2001-026.

9. J. Charles, Phys. Rev. D59, 054007 (1999).

10. M. Ciuchini et al., JHEP 0107 (2001) 013.

11. D. E. Groom et al., Eur. Phys. Jour. C15, 1 (2000).

12. The LEP B Oscillation Working Group, http://lepbosc.web.cern.ch/LEPBOSC. 